\title{
Correction to: Let's Build a Multiplayer Phaser Game
}

\section{Correction to:}

O. Lodriguez, Let's Build a Multiplayer Phaser Game, https://doi.org/10.1007/978-1-4842-4249-0

The text highlighted occurring in listing 4-16 in Chapter 4 mentioned below: keyboard.model.ts

Has been replaced with:

\section{keyboard.class.ts}

The text highlighted occurring in listing 5-2 in Chapter 5 mentioned below: player.class.ts

Has been replaced with:

game.class.ts

The above mentioned corrections have been updated in the book.

\footnotetext{
The updated versions of the chapters could be found at https://doi.org/10.1007/978-1-4842-4249-0_4 https://doi.org/10.1007/978-1-4842-4249-0_5 